\title{
Thirty One
}

National Cancer Institute

\section{Source}

National Cancer Institute. Thirty One. NCI Thesaurus. Code C126863.

A natural number greater than thirty and less than thirty-two and the quantity that it denotes. 